\title{
Ajuste de fármacos y nutrición en terapias de reemplazo renal continuo
}

\author{
Jorge Echeverri' y Alejandra Molano²
}

${ }^{1}$ Servicio de Nefrología Hospital Militar Central, Facultad de Medicina de la Universidad Militar Nueva Granada; ${ }^{2}$ Servicio de Nefrología Fundación Cardio Infantil, Facultad de Medicina de la Universidad del Rosario, Bogotá. Colombia

\begin{abstract}
Resumen
Del 6 al 23\% de los pacientes con lesión renal aguda (LRA) en unidades de cuidados intensivos (UCI) requieren apoyo renal, siendo la terapia continua una modalidad de alta frecuencia de uso en el paciente críticamente enfermo. Si bien el objetivo general de las terapias de reemplazo renal continuo (TRRC) es restablecer el equilibrio hídrico y ácido-base, junto con la eliminación de toxinas urémicas e inflamatorias relacionadas con la pérdida de depuración renal y la disfunción multiorgánica; reconocemos como efecto colateral la depuración $(K)$ no deseado de moléculas y sustancias deseadas en la recuperación del paciente crítico, como pueden ser antimicrobianos y nutrientes. La sepsis es la causa más frecuente de LRA en la UCl y en este contexto la terapia antimicrobiana adecuadamente seleccionada y a la dosis correcta es la médica terapéutica más importante. De la misma manera, es indispensable garantizar el adecuado apoyo nutricional en este grupo poblacional. Proponemos en esta revisión una aproximación teórica y práctica para seleccionar el tratamiento farmacológico de antimicrobianos y el apoyo nutricional en el paciente en TRRC.
\end{abstract}

PALABRAS CLAVE: Dosis. Terapéutica. Extracorpórea. Eliminación. Nutrición. Hemofiltración.

\begin{abstract}
Six to $23 \%$ of patients with acute kidney injury (AKI) in intensive care units (ICU) require renal support. Continuous renal replacement therapies (CRRT) have become the modality of choice in critical care. Although the aim of CRRT is to restore the water and acid-base balance, together with the removal of uremic and inflammatory toxins related to the loss of renal clearance and multi-organ dysfunction; we recognize as a side effect the unwanted clearance of molecules and substances desired for the recovery of the critically ill patient such as antimicrobials and nutrients. Sepsis is the most frequent cause of AKI in the ICU and, in this context, the appropriate selection of antimicrobial therapy, and at the correct dose, is one of the most important decisions; it is also essential to guarantee the adequate nutritional support in this population. We propose in this review a theoretical and practical approach to address the pharmacological management of antimicrobials and nutritional support in the patient in CRRT.
\end{abstract}

KEY WORDS: Dose. Therapeutical. Extracorporeal. Elimination. Nutrition. Hemofiltration.

Correspondencia:

Jorge Echeverri

E-mail: je.echeverri.s@gmail.com
Fecha de recepción: 16-11-2017

Fecha de aceptación: 08-03-2018

DOI: 10.24875/GMM.M18000065
Gac Med Mex. 2018;Supp 1:48-60

Disponible en PubMed www.gacetamedicademexico.com 


\section{Introducción}

El ajuste de medicamentos en el paciente con LRA constituye una prioridad para el clínico con el fin de garantizar lograr los objetivos terapéuticos propuestos, sin embargo, la información respecto al empleo individualizado de cada grupo farmacológico es limitada'. Dada la alta prevalencia de la sepsis y la LRA vamos a centrar la revisión en el grupo farmacológico de antibióticos, donde puede estar la información más relevante.

La causa más frecuente de LRA en los pacientes en $\mathrm{UCl}$ es la sepsis, siendo más del $50 \%$ de los casos de LRA; hasta el $37 \%$ de los pacientes sépticos presenta LRA en algún momento de su evolución en la UCl2,3. La inadecuada selección del antibiótico, el retraso en el inicio de su administración y las dosis inapropiadas que no cumplen con los objetivos farmacocinéticos y farmacodinámicos tienen un significativo impacto en los resultados de pacientes con sepsis y choque séptico, aumentando de manera notoria la mortalidad intrahospitalaria ${ }^{3,4}$. Los cambios fisiopatológicos de los pacientes en $\mathrm{UCl}$ con LRA afectan la farmacocinética y farmacodinámica de los medicamentos, siendo usualmente difícil predecir sus efectos; específicamente en el caso de los antimicrobianos, no solo alteran su acción bactericida, sino que pueden facilitar la aparición de resistencia ${ }^{5}$.

Para comprender mejor la farmacología en los pacientes con LRA es pertinente recordar los conceptos generales de la acción antimicrobiana. El proceso de absorción, distribución, metabolismo y eliminación se denomina farmacocinética; y el estudio del mecanismo de acción en relación con la concentración del medicamento y su efecto en el organismo se llama farmacodinámica.

\section{Generalidades de antimicrobianos}

Antes de analizar cómo interpretar las variaciones farmacocinéticas de los antimicrobianos en los pacientes críticos con LRA en TRRC y su aplicación en el ajuste de la dosificación de los antibióticos, es importante entender los objetivos farmacodinámicos de los antimicrobianos. Se reconoce en la actualidad que el éxito terapéutico depende altamente de las concentraciones alcanzadas en el sitio de infección, sabiéndose que los efectos de los antibióticos están ligados a la exposición para su actividad bactericida, concretamente en relación con la concentración mínima inhibitoria (CIM). La mayoría de los antibióticos pueden ser clasificados en tres grupos farmacodinámicos: dependientes del tiempo (tiempo sobre la CIM), dependientes de la concentración (concentración máxima sobre la CIM) y dependientes de la concentración y el tiempo (área bajo la curva $[\mathrm{AUC}]$ sobre la $\mathrm{CIM})^{6,7}$ (Fig. 1 y Tabla 1).

\section{Antimicrobianos dependientes de la concentración}

Este grupo logra su eficacia bactericida al alcanzar una concentración pico máxima 8 a 10 veces la CIM, seguido por un valle bajo para minimizar los efectos adversos, lo cual determina el "efecto postantibiótico», que se refiere al tiempo que se requiere para que el microorganismo recupere el crecimiento normal después de la exposición al agente antimicrobiano; ambos mecanismos permiten tener un intervalo de dosificación amplio. Es usual el uso de dosis de carga (DC) para alcanzar una adecuada concentración, en especial en los pacientes críticos que experimentan incrementos de los volúmenes de distribución de algunos de estos medicamentos ${ }^{8,9}$.

\section{Antimicrobianos dependientes del tiempo}

Este grupo requiere del mantenimiento de concentraciones óptimas sobre la CIM para tener el efecto bactericida y evitar la resistencia ( $>40 \%$ tiempo sobre la CIM para carbapenémicos $0>70 \%$ del tiempo sobre la CIM para cefalosporinas). En este caso, por más que se aumente la concentración del antibiótico, el efecto bactericida no aumenta (efecto bactericida de orden cero). En general, el máximo efecto antimicrobiano se logra al alcanzar niveles 4 a 5 veces la CIM. Usualmente se ha preferido el método de administración continua para lograr garantizar mantener los niveles en el objetivo terapéutico en la mayor cantidad del tiempo $0^{10,11}$.

\section{Antimicrobianos dependientes del tiempo y la concentración}

Este grupo alcanza su eficacia farmacológica dependiendo de la exposición total por encima de la CIM al antimicrobiano; se utiliza el AUC sobre la CIM como parámetro de cuantificación y la meta es fijada de forma individual por antimicrobiano ${ }^{12}$.

$$
\text { AUC/CIM= Concentración (C) x Tiempo (T)/CIM }
$$

\section{Farmacocinética de antimicrobianos en la lesión renal aguda}

\section{Absorción y biodisponibilidad}

La absorción es el movimiento del medicamento del sitio de administración al compartimento central. De 


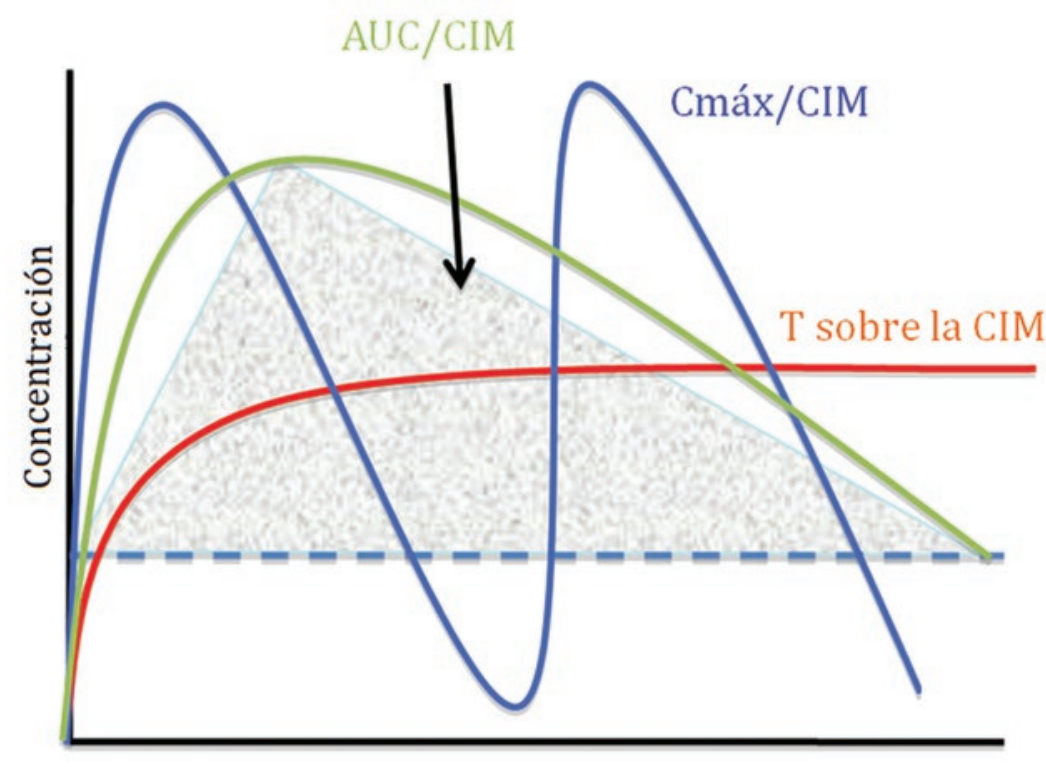

Tiempo

Figura 1. Grupos de antimicrobianos según su mecanismo farmacodinámico.

T sobre la CIM: tiempo de la concentración libre del fármaco por encima de la concentración inhibitoria mínima durante el intervalo de dosificación; Cmáx/CIM: relación entre la concentración libre del fármaco y la concentración inhibitoria mínima; AUC/CIM: exposición a la concentración libre del fármaco por encima de la concentración inhibitoria mínima durante un periodo de 24 horas.

Tabla 1. Parámetros farmacodinámicos de algunos antimicrobianos

\begin{tabular}{ll}
\hline Antibiótico & Medida farmacodinámica \\
\hline Dependiente de la concentración & \\
Metronidazol & Concentración pico-CIM \\
Colistina & AUC/CIM \\
Aminoglucósidos & AUC/CIM, concentración pico-CIM \\
Daptomicina & AUC/CIM, concentración pico-CIM \\
Fluoroquinolonas & AUC/CIM, concentración pico-CIM \\
Equinocandinas & AUC/CIM, concentración pico-CIM \\
Macrólidos & AUC/CIM \\
Dependiente del tiempo & \\
Betalactámicos: penicilinas, cefalosporinas, carbapenémicos & \\
Glucopéptidos & Tiempo/CIM \\
Aztreonam & AUC/CIM \\
Clindamicina & Tiempo/CIM \\
Tetraciclinas & AUC/CIM \\
Linezolida & AUC/CIM \\
\hline AUC:árobio lacla & AUC/CIM \\
\hline
\end{tabular}

AUC: área bajo la curva; CIM: concentración inhibitoria mínima.

este concepto se deriva el de biodisponibilidad $(F)$ que se refiere a la fracción del medicamento absorbido que llega a la circulación sin cambios. En general los medicamentos administrados por vía endovenosa suelen tener una biodisponibilidad de 1 ( $F$ de 1.0) a menos que sean profármacos. La biodisponibilidad menor a 1 se correlaciona con disminución de la absorción o es secundaria a metabolismo presistémico, 
usual en los medicamentos de administración enteral.

La absorción de medicamentos orales en los pacientes críticamente enfermos se altera por el edema de la pared intestinal y por la dismotilidad, condiciones acentuadas en los que tienen tratamiento vasopresor en relación con la hipoperfusión ${ }^{13,14}$. Con la uremia se puede ver afectada la absorción de algunos medicamentos, encontrándose en experimentos animales inhibición del efecto de metabolismo de primer paso y de la absorción gastrointestinal. De igual forma, el uso de profilaxis gástrica, quelantes y resinas pueden retardar la absorción de algunos antibióticos ${ }^{15}$. El efecto de primer paso puede aumentar o disminuir la $F$, tal es el caso de la disminución de la biodisponibilidad de los profármacos en los pacientes con disfunción hepática severa. La nutrición enteral en preparados comerciales puede disminuir la absorción de ciertos medicamentos, como es el caso del ciprofloxacino oral ${ }^{16}$.

\section{Distribución}

Después de la absorción o la administración sistémica al torrente sanguíneo, el medicamento se distribuye en el espacio intersticial e intracelular. Este proceso depende de las características fisicoquímicas propias de cada medicamento y las condiciones del paciente como el gasto cardíaco, el flujo sanguíneo regional, la permeabilidad capilar y el volumen de tejido perfundido ${ }^{17}$.

El volumen de distribución $(\mathrm{Vd})$ de un medicamento es equivalente a la cantidad de este medicamento en el cuerpo (dosis) con relación a su concentración plasmática en estado estable. En los pacientes con sepsis, el daño endotelial y del glucocálix causa un aumento de la permeabilidad capilar, lo cual se traduce en la salida de líquido del espacio intravascular hacia el intersticio. Adicionalmente, por la administración no controlada de líquidos y la oliguria en la LRA es frecuente encontrar balances hídricos positivos ${ }^{18}$, lo que ocasiona aumento del Vd de medicamentos hidrofílicos, como los aminoglucósidos, los betalactámicos y los glucopéptidos; encontrando en consecuencia disminución de las concentraciones sanguíneas de estos ${ }^{19}$ (Tabla 2).

Por el contrario, los medicamentos lipofílicos (p. ej., quinolonas, tigeciclina), se distribuyen en gran extensión intracelularmente y en el tejido adiposo, de tal manera que en los pacientes obesos los antimicrobianos lipofílicos generalmente tienen un gran $\mathrm{Vd}$ y no son influenciados por el movimiento de líquidos en el paciente con LRA ${ }^{20}$. El grado de obesidad del paciente es relevante en medicamentos hidrofílicos con alto $\mathrm{Vd}$, en donde es necesario realizar ajustes en los esquemas de dosificación para evitar fallo terapéutico ${ }^{21}$. De tal manera que algunos antibióticos como piperacilina/ tazobactam y doripenem requieren altas dosis e infusiones extendidas para lograr adecuada exposición del medicamento, en estas condiciones ${ }^{22,23}$.

Muchos de los procesos infecciosos que ocurren en el intersticio tisular suelen tener alteraciones en su microcirculación, causando una especie de "compartimentalización" que retrasa la adecuada concentración de antimicrobianos. Solamente cuando se tiene un equilibrio en la distribución (entrada y salida del medicamento

Tabla 2. Cambios en las propiedades farmacocinéticas de antimicrobianos en el paciente crítico

\begin{tabular}{|c|c|c|}
\hline Grupo antimicrobiano & Farmacocinética usual & $\begin{array}{c}\text { Farmacocinética en paciente crítico } \\
\text { y lesión renal aguda }\end{array}$ \\
\hline $\begin{array}{l}\text { Hidrofílicos (betalactámicos, carbapenémicos } \\
\text { aminoglucósidos, glucopéptidos, linezolida, colistina, } \\
\text { fluconazol, daptomicina) }\end{array}$ & $\begin{array}{c}\text { Bajo Vd } \\
\text { Baja penetración intracelular } \\
\text { Aclaramiento renal predominante }\end{array}$ & $\begin{array}{c}\text { Aumento Vd } \\
\text { Disminuye penetración intracelular }\end{array}$ \\
\hline $\begin{array}{l}\text { Lipofílicos (fluoroquinolonas, macrólidos, } \\
\text { lincosamidas, tigeciclina) }\end{array}$ & $\begin{array}{c}\text { Alto Vd } \\
\text { Alta penetración intracelular } \\
\text { Aclaramiento hepático } \\
\text { predominante }\end{array}$ & $\begin{array}{l}\text { Alto Vd } \\
\text { Aclaramiento hepático predominante }\end{array}$ \\
\hline \multicolumn{3}{|l|}{ Vd en antimicrobianos de alto uso en cuidado crítico } \\
\hline Piperacilina ${ }^{26,27}$ & 0.15 & 0.18 \\
\hline Meropenem ${ }^{28,29}$ & 0.17 & 0.37 \\
\hline Amikacina ${ }^{30,31}$ & 0.18 & 0.44 \\
\hline Vancomicina ${ }^{32,33}$ & 0.39 & 0.65 \\
\hline Daptomicina ${ }^{34,35}$ & 0.1 & 0.23 \\
\hline
\end{tabular}


a una tasa equivalente) la concentración sanguínea del medicamento representa el Vd. Por esta razón, un valor único adecuado de concentración sérica del medicamento no necesariamente garantiza una buena biodisponibilidad en el tejido comprometido por la infección ${ }^{24}$.

La mayoría de los medicamentos son ácidos o bases orgánicas débiles en forma ionizada o no ionizada en medios acuosos. El pKa es el pH en el cual las concentraciones ionizadas y no ionizadas de la sustancia son equivalentes. Cuando el pH es menor que el pKa, la forma no ionizada de un ácido débil aumenta, pero la forma ionizada de una base débil predomina, mejorando su capacidad hidrofílica. La acidemia, frecuente en la sepsis y la LRA, favorece un aumento en la forma no ionizada de los ácidos débiles, facilitando el paso a través de las membranas celulares y aumentando el $\mathrm{Vd}$ de la sustancia. Se han descrito cambios en el Vd en presencia de ventilación mecánica y circuitos extracorpóreos ${ }^{25}$.

El Vd de un medicamento permite conocer la DC de este para lograr una concentración plasmática deseada, siendo la DC igual a la concentración de un medicamento deseado multiplicado por su Vd en un estado estable ${ }^{8}$.

Dosis de carga $=$ Concentración $\mathrm{x}$ Volumen de distribución

\section{Unión a proteínas plasmáticas}

La hipoalbuminemia es una condición usual de los pacientes en cuidado intensivo con LRA, puede presentarse entre el 40 a $50 \%$ de los casos, básicamente por el efecto de reactante negativo de fase aguda, pérdida transcapilar por disfunción endotelial y dilución por sobrecarga de líquidos. Los efectos de la hipoalbuminemia en la farmacocinética están relacionados el aumento de la fracción libre del fármaco, incremento de su Vd y aumento del aclaramiento del plasma. No existen reglas específicas de dosificación en el contexto de hipoalbuminemia, pero este es un aspecto a considerar en pacientes con albúmina sérica menor de 2,5 g/dl y uso de antibióticos de amplia unión a proteínas (p. ej., goxacilina, ceftriaxona, clindamicina, ertapenem, daptomicina); requiriéndose incremento de dosis totales, infusiones continuas o extendidas para lograr una óptima exposición al fármaco ${ }^{36,37}$.

\section{Eliminación}

La eliminación de los antibióticos en pacientes con LRA en UCl depende de la eliminación no renal (hígado, piel, pulmón), eliminación renal residual y el aclaramiento por terapias de reemplazo renal (TRR). La mayoría de los antibióticos siguen el modelo cinético de eliminación de primer orden, en el que la tasa de eliminación es proporcional a la cantidad del medicamento en el cuerpo ${ }^{38,39}$.

La capacidad de aclaramiento está determinada por el tamaño de la sustancia, el Vd y la capacidad de unión a proteínas. La LRA se caracteriza no solamente por disminución aguda de la tasa de filtración glomerular, sino también por disfunción tubular, la cual puede ser aún más relevante para los procesos de secreción y reabsorción tubular. Los betalactámicos son por excelencia el ejemplo de moléculas de amplia unión a proteínas que requiere de la secreción tubular proximal para su eliminación renal ${ }^{40}$.

El ajuste de la dosis en los pacientes con LRA ha tenido como intención evitar la sobredosificación de antimicrobianos, especialmente en población en TRR; utilizando frecuentemente los modelos farmacológicos de pacientes con compromiso renal crónico y en el contexto fuera de cuidado intensivo ${ }^{41,42}$.

Estudios de evaluación farmacológica en pacientes en TRR en UCI nos muestran que una población importante puede estar subdosificada, dado que se ha documentado que entre el 25 y el $60 \%$ tienen niveles de antibióticos subterapéuticos ${ }^{43,44}$. Es por eso por lo que consideramos importante integrar los principios del aclaramiento de las TRRC junto con los conceptos farmacocinéticos de los antimicrobianos; esto con el fin de que el clínico mejore sus herramientas de decisión en el cálculo de las dosificaciones ajustadas a las condiciones de cada paciente, sin incrementar el riesgo de toxicidad y minimizando la probabilidad de fracaso terapéutico ${ }^{45}$.

\section{Terapias de reemplazo renal continuo y antimicrobianos}

\section{Conceptos generales en depuración continuo}

Existen dos principios básicos para aclaramiento $(\mathrm{K})$ de sustancias en las TRR. El primero de ellos es la difusión, característico de las técnicas de hemodiálisis venovenosa continua (HDVVC) en la cual, gracias a un gradiente de concentración, una molécula atraviesa una membrana semipermeable. El segundo principio físico es la convección, en el cual el arrastre de agua por diferencia de presión causa desplazamiento de moléculas a través de una membrana, como es el caso de la hemofiltración venovenosa continua (HFVVC). La 
convección característicamente permite la eliminación de partículas de mayor tamaño que tienen menor capacidad cinética y por tanto más difícil es el paso de la membrana por diferencia de concentración. Los antibióticos que tienen gran $\mathrm{Vd}$ pueden ser mejor eliminados por técnicas de difusión, simplemente por la presencia de un continuo gradiente entre el plasma y los demás compartimentos. La mezcla de ambas propiedades da origen a la terapia de hemodiafiltración venovenosa continua (HDFVVC). La eliminación efectiva de una molécula depende adicionalmente de otras variables como son las características de la membrana, su permeabilidad molecular o coeficiente de eliminación (cribado, Sc), su grado de absorción/adsorción, el efecto de polarización de membrana (Gibs-Donan), los esquemas de reposición de líquidos (predilución y posdilución) y la dosis de TRRC entre otras ${ }^{46}$.

(1) Sc = Concentración en efluente (Cef)/Concentración en plasma $(\mathrm{Cp})$

(2) $\mathrm{K}=$ Dosis TRRC $\times \mathrm{Sc}$

Los principios físicos aplicados han sido posibles gracias al desarrollo de las diferentes membranas de los filtros. Las membranas de TRRC actuales tienen la suficiente permeabilidad como para permitir el paso los antibióticos, lo que corresponde a un Sc in vitro de 1 (permite el paso completo de la molécula). Todos los antibióticos comercialmente disponibles tienen un peso molecular menor de 2,000 DA; por tanto, la unión a proteínas, la interacción de las moléculas con la membrana del filtro (Efecto Gibs Donan en aminoglucósidos y ceftazidima) y su Vd son los determinantes principales del aclaramiento de los antibióticos. Cuando se usa predilución, hay que corregir la dosis real aportada de hemofiltración a un factor de corrección (Fc), por el efecto de dilución del plasma antes de entrar al dializador.

(3) Fc = Flujo de sangre $(Q b) /(Q b+$ Flujo de reposición (Qr))

\section{¿Como calcular la dosis de un antibiótico?}

Las prácticas actuales en TRRC se caracterizan por una amplia variabilidad en su ejecución al lado de la cama del paciente crítico, diferentes momentos de inicio, diversidad en los esquemas de dosificación alcanzados, requerimientos de ultrafiltración variable, contextos clínicos y estados hídricos muy diferentes entre cada uno de los enfermos intervenidos; situaciones que hacen importante poder tener una aproximación individualizada de dosificación de antimicrobianos mientras logramos obtener una monitorización más estrecha farmacológica y recomendaciones fuertes basadas en la evidencia científicas. Conocer el Vd, la CIM deseada del antibiótico para el germen respectivo, el Sc del antibiótico para la membrana y la cuantificación exacta del aclaramiento renal residual pueden darnos herramientas prácticas para definir en el paciente en TRRC tanto las dosis iniciales del antimicrobiano como las dosis de mantenimiento (DM).

Inicialmente debemos calcular la DC basándonos en el Vd y la concentración deseada en relación con la CIM del microorganismo. La DM depende de las características farmacodinámicas del medicamento, el aclaramiento renal, no renal y de la terapia extracorpórea. Las especificaciones de cada antibiótico están descritas en la tabla 3.

- Ejemplo 1: Antimicrobiano dependiente de la concentración

Un paciente que tiene una bacteriemia por bacilos gramnegativos y se selecciona un tratamiento con amikacina. Su peso es de $60 \mathrm{~kg}$ y tiene LRA estadio 3. Está anúrico, en terapia con HFVVC a dosis de $35 \mathrm{ml} / \mathrm{kg} / \mathrm{hora} 70 \%$ en predilución, la bomba de sangre programada es de $200 \mathrm{ml} / \mathrm{min}$. La CIM de la amikacina para el germen es de $3 \mathrm{mg} / \mathrm{l}$.

Dosis de carga:

La DC, dependiente del Vd, es igual a la concentración deseada de 8 veces la CIM para la amikacina $(8 \times 3 \mathrm{mg} / \mathrm{l}) \times$ el Vd (que se modifica con LRA de 0.18 a $0.44 \mathrm{l} / \mathrm{kg}$ ), lo cual equivale a $633 \mathrm{mg}$ de amikacina de DC.

$$
\begin{aligned}
& \mathrm{DC}=8 \times \mathrm{CIM} \times \mathrm{Vd} \\
& \mathrm{DC}=8 \times 3_{\mathrm{mg} / /} \times 0.44_{1 / \mathrm{kg}} \times 60 \mathrm{~kg} \\
& \mathrm{DC}=633 \mathrm{mg} \text { para una concentración en sangre de }
\end{aligned}
$$
$24 \mathrm{mg} / \mathrm{l}$

Dosis de mantenimiento:

La DM de la amikacina es dependiente del aclaramiento. La amikacina en un paciente anúrico tiene UN aclaramiento no aportado por la terapia extracorpórea que es de $23 \mathrm{ml} / \mathrm{min}$ (hepático, piel y otros tejidos), más el efecto de la HFVVC: flujo de ultrafiltración por el factor de corrección (predilución), multiplicado por el coeficiente de cribado de la molécula. Así, el aclaramiento por la HVVC es de 2,100 (peso x $35 \mathrm{ml} / \mathrm{kg} / \mathrm{h}$ ) x Fc (0.9) x Sc (0.6 para la amikacina) que es igual a $18.9 \mathrm{ml} / \mathrm{min}$; es decir, $41.9 \mathrm{ml} /$ min al sumar el efecto no aportado por la TRR.

$$
\begin{aligned}
& \text { Aclaramiento }(\mathrm{K}) \mathrm{HVVC}=\text { Quf } \times \mathrm{Fc} \times \mathrm{Sc} \\
& \mathrm{K}=2,100 \times 0.9 \times 0.6=18.9_{\mathrm{m} / \mathrm{min}} \\
& \mathrm{K} \text { total }=18.9_{\mathrm{m} / \mathrm{min}}+23_{\mathrm{m} / \mathrm{min}}=41.9_{\mathrm{m} / \mathrm{min}}
\end{aligned}
$$

La amikacina es un antibiótico considerado dependiente de la concentración sobre la CIM, por lo cual es indispensable calcular la vida media. El T vida media 


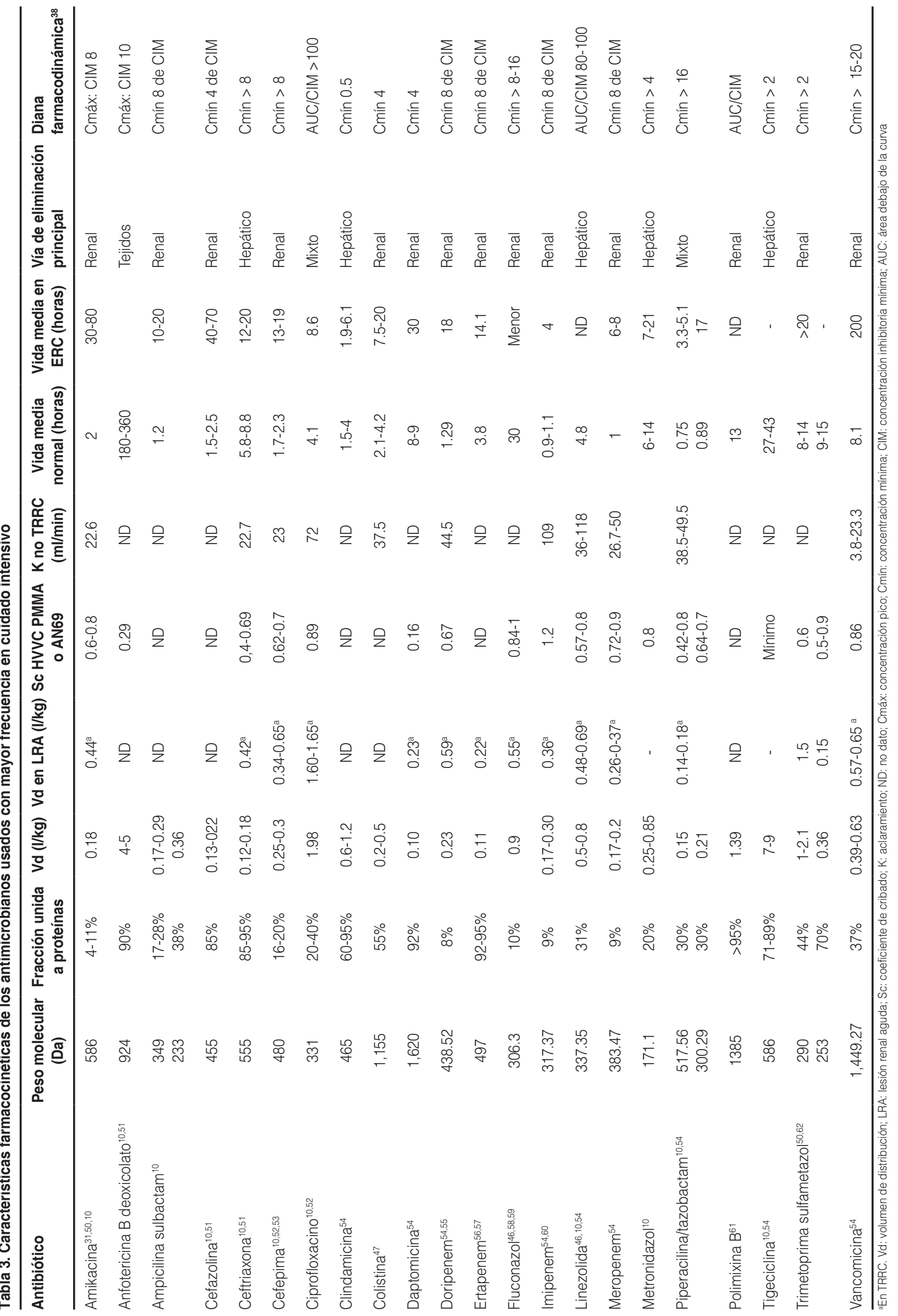


es la relación entre el producto 0.693 por el Vd entre el aclaramiento del medicamento. Para este paciente, el cálculo de la vida media es de 7.27 horas. Calculando la concentración valle menor de $1 \mathrm{mg} / \mathrm{l}$, se requieren al menos 5 vidas medias para bajar la concentración de 24 a $1 \mathrm{mg} / \mathrm{l}$.

$$
\begin{aligned}
& \mathrm{T} 1 / 2=(0.693 \times \mathrm{Vd}) \div \text { Aclaramiento } \\
& \mathrm{T} 1 / 2=\left(0.693 \times 26,400_{\mathrm{ml}}\right) \div 41.9_{\mathrm{m} / \mathrm{min}} \\
& \mathrm{T} 1 / 2=436 \mathrm{~min}=7.27 \text { horas } \\
& \mathrm{DM}=7.27 \text { horas } \times 5=36 \text { horas }
\end{aligned}
$$

La dosis recomendada para este paciente anúrico en HFVVC es amikacina $633 \mathrm{mg}$ cada 36 horas y no $5-7.5 \mathrm{mg} / \mathrm{kg}$ cada 48 horas, como está recomendado en algunos libros de ajustes de dosis.

- Ejemplo 2: Antimicrobiano dependiente del tiempo

Un paciente con sepsis abdominal por una perforación intestinal está en tratamiento antibiótico con meropenem. Después de la intervención quirúrgica, el paciente regresa a la $\mathrm{UCl}$ con alto soporte vasopresor, anúrico y requiriendo inicio de HDFVVC. Tiene un peso de ingreso de $65 \mathrm{~kg}$ y actual de $70 \mathrm{~kg}$ en relación con edemas en extremidades y declive. El médico le prescribe una dosis total de efluente de $30 \mathrm{ml} / \mathrm{kg} / \mathrm{h}$ en posfiltro con $25 \%$ de dosis de diálisis y ordena una extracción por hora adicional de $300 \mathrm{cc}$ para dejar en balance negativo. ¿Cómo ajustaría la dosis de meropenem en este paciente?

Los estudios demuestran un Vd del meropenem en el paciente crítico oscila entre $0.26 \mathrm{l} / \mathrm{kg}$ a $0.38 \mathrm{l} / \mathrm{kg}$. Entonces se calcula la DC para tener una concentración de meropenem de $20 \mathrm{mg} / \mathrm{l}$ la cual garantiza una buena disponibilidad intrabdominal y un $\mathrm{Vd}$ aproximado de $0.30 \mathrm{l} / \mathrm{kg}$

Dosis de Carga:

$$
\begin{aligned}
& D C=\text { Concentración sérica } \times \mathrm{Vd} \\
& \quad \mathrm{Vd}=0.30_{\mathrm{l} / \mathrm{kg}} \times 65_{\mathrm{kg}}+5_{\mathrm{lt}} \text { de edemas } \\
& \quad \mathrm{Vd}=24.5_{\mathrm{l}} \\
& \mathrm{DC}=20_{\mathrm{mg} / \mathrm{l}} \times 24.5_{\mathrm{l}} \\
& \mathrm{DC}=490_{\mathrm{mg}}
\end{aligned}
$$

Dosis de mantenimiento:

Luego, para calcular la DM igual que para la vancomicina se calcula la eliminación total del medicamento. El aclaramiento no renal del meropenem está alrededor de $30 \mathrm{ml} / \mathrm{min}$. El aclaramiento por la TRR es:

$\mathrm{K}$ de la TRR es = Flujo de efluente $\mathrm{x}$ Sc

$\mathrm{K} \mathrm{TRR}=\left(65_{\mathrm{kg}} \times 30_{\mathrm{m} / \mathrm{kg} / \mathrm{h}}+300_{\mathrm{m} / \mathrm{h}}\right.$ de extracción $) \times 0.9$ $\mathrm{K}$ TRR $=2,250 \mathrm{cc} \times 0.9=33.7_{\mathrm{m} / \mathrm{min}}$

$\mathrm{K}$ total $=\mathrm{K}$ renal $+\mathrm{K}$ no renal $+\mathrm{K}$ TRRC $=0+$ $30_{\mathrm{m} / \mathrm{min}}+33.7_{\mathrm{m} / \mathrm{min}}$

$\mathrm{K}$ total $=63.7_{\mathrm{m} / \mathrm{min}}$

Como este antibiótico es dependiente del tiempo, la dosis es igual a la tasa de eliminación.

$$
\mathrm{DM}=\text { Concentración } \times \mathrm{K}_{\text {Total }}
$$$$
\mathrm{DM}=20_{\mathrm{mg} / 1} \times 63.7_{\mathrm{m} / \mathrm{min}}
$$

$$
\begin{aligned}
& \mathrm{DM}=20_{\mathrm{mg} / /} \times 0.0637_{\mathrm{I} / \mathrm{min}} \\
& \mathrm{DM}=1.2_{\mathrm{mg} / \mathrm{min}} \\
& \mathrm{DM}=1,728_{\mathrm{mg} \text { en } 24 \text { horas infusión continua }}
\end{aligned}
$$

Entonces, se puede indicar una infusión de 1,728 mg en 24 horas. Considerando el rango de seguridad que se tiene con este antibiótico y la baja posibilidad de tener niveles disponibles en la clínica se recomienda usar hasta el $25 \%$ más de la dosis calculada.

- Ejemplo 3: Antimicrobiano dependiente del tiempo y la concentración

Se necesita tratar una bacteriemia por Enterococcus faecium resistente a ampicilina en un paciente hombre de $70 \mathrm{~kg}$ de peso con LRA estadio 3 y uresis residual que otorga una depuración de creatinina de $12 \mathrm{ml} / \mathrm{min}$ en orina de 4 horas. El paciente se encuentra en TRRC en modalidad de hemodiafiltración a dosis de 2 I/hora en posdilución y un baño de diálisis de $1 \mathrm{l} / \mathrm{hora}$.

¿Cuál es la DC y de mantenimiento de la vancomicina en este paciente para tener una concentración de $20 \mathrm{mg} / \mathrm{l}$ si se considera que la vancomicina tiene en estos casos un $\mathrm{Vd}$ de $0.6 \mathrm{l} / \mathrm{kg}$ ?

Dosis de carga:

$\mathrm{DC}=$ Concentración sérica $\mathrm{x} \mathrm{Vd}$

$\mathrm{DC}=20_{\mathrm{mg} / \mathrm{l}} \times 0.6_{1 / \mathrm{kg}} \times 70_{\mathrm{kg}}$

$\mathrm{DC}=840_{\mathrm{mg}}$

Dosis de mantenimiento:

La DM se determina una vez se obtenga el aclaramiento por la TRR más el aporte de depuración renal residual de la vancomicina.

$\mathrm{K}$ de la TRR es = Flujo de efluente $\mathrm{x}$ Sc

El Sc de la vancomicina conocido para algunos filtros es alrededor de 0.7 .

$$
\begin{aligned}
& \mathrm{K} \text { TRRC }=3 \mathrm{l} / \mathrm{h}(2 \text { de HF y } 1 \text { de HD) } \times 0.7 \\
& \mathrm{K} \text { TRRC }=35_{\mathrm{m} / \mathrm{min}} \\
& \mathrm{K} \text { total }=\mathrm{K} \text { renal }+\mathrm{K} \text { no renal }+\mathrm{K} \text { TRRC }=12_{\mathrm{m} / \mathrm{min}}+ \\
& 3.8_{\mathrm{m} / \mathrm{min}}+35_{\mathrm{m} / \mathrm{min}} \\
& \mathrm{K} \text { total }=50.8_{\mathrm{m} / / \mathrm{min}} \\
& \text { Si la recomendación es aplicar infusión de vancomicina, }
\end{aligned}
$$
entonces la DM es igual a la de eliminación. Para lograr una concentración estable de $20 \mathrm{mg} / \mathrm{l}$ se requiere multiplicar $20 \mathrm{mg} / \mathrm{l} x$ depuración total $(50.8 \mathrm{ml} / \mathrm{min}$ ) equivalente a $0.94 \mathrm{mg} / \mathrm{min}$ o $56 \mathrm{mg} / \mathrm{hora}=1,344 \mathrm{mg}$ cada 24 horas.

$\mathrm{DM}=$ Concentración $\times$ Depuración total

$\mathrm{DM}=20_{\mathrm{mg} / \mathrm{l}} \times 50.8_{\mathrm{m} / \mathrm{min}}$

$\mathrm{DM}=1,016_{\mathrm{mg} / \mathrm{min}}=69,96_{\mathrm{mg} / \mathrm{h}}$

$\mathrm{DM}=1463_{\mathrm{mg} \mathrm{c} / 24} \mathrm{~h}$ infusión continua

En estos medicamentos en los cuales la ventana terapéutica es tan estrecha, la recomendación es medir concentración continuamente. Un experimento individual en 10 pacientes en terapia de HDFVVC a dosis de $3 \mathrm{l} / \mathrm{h}$ (predilución $2 \mathrm{l} / \mathrm{h}$ y $1 \mathrm{l} / \mathrm{hora}$ de diálisis) muestra resultados similares con indicación de aplicación de 450 mg 
cada 12 horas en pacientes anúricos para mantener una concentración de $15 \mathrm{mg} / \mathrm{l}$ estable. Muy similar a lo indicado realizando estos cálculos ${ }^{33}$.

En la actualidad existen propuestas en investigación multicéntrica encaminadas a mejorar nuestro conocimiento del comportamiento farmacocinético de los antimicrobianos en TRRC, evaluando diferentes escenarios en el cuidado intensivo y la influencia de diferentes variables dinámicas del paciente crítico ${ }^{48,49}$. En la tabla 3 presentamos al lector información de interés de diferentes antimicrobianos, según la literatura actual disponible, con respecto a variables farmacocinéticas y de aclaramiento, con el fin de facilitar la aplicación de los cálculos expuestos e individualizar los esquemas de dosificación en TRRC.

\section{Terapias nutricionales en la TRRC}

La terapia nutricional constituye uno de los elementos básicos del tratamiento integral de los pacientes en unidades de cuidado crítico, sin embargo, existen carencias en el cuidado de este componente, como ha sido plasmado por grupos como el de The Nutrition Day ICU survey, con observaciones de siete años en pacientes de $880 \mathrm{UCl}$ de 46 países $^{63}$.

El grupo de Bendavida, et al. encontró que frecuentemente los pacientes no reciben las cantidades recomendadas de calorías y proteínas, describiéndose que un bajo aporte nutricional puede relacionarse con aumento de tiempos de permanencia en $\mathrm{UCl}$, lo que a su vez incrementa el riesgo de infecciones y mortalidad; en contraposición, el aporte energético excesivo se asocia con complicaciones y mortalidad ${ }^{63}$.

Los pacientes en $\mathrm{UCl}$ implican un gran reto terapéutico desde el punto de vista nutricional, dada su alta diversidad, que oscila entre la caquexia y la obesidad mórbida ${ }^{64,65}$.

En el contexto de los pacientes con LRA y específicamente recibiendo TRRC, hay características particulares:

- El uso de TRRC modifican el balance de líquidos de los pacientes, con lo que se obtienen rangos amplios de osmolaridad y volemia.

- La TRRC puede reducir la formación de edema de la pared intestinal (frecuente en estados post reanimación), lo que resulta de utilidad en el inicio temprano (antes de $48 \mathrm{~h}$ ) de la nutrición enteral sugeridas por la mayoría de las guías de sociedades científicas (European Society for Clinical Nutrition and Metabolism [ESPEN], American Society for Parenteral and Enteral Nutrition, International Symposium on Intensive Care and
Emergency Medicine) permitiendo la absorción de nutrientes administrados enteralmente ${ }^{64,66}$.

- Sin embargo, la mayoría de los nutrientes tiene un peso molecular que los hace susceptibles de ser eliminados por los filtros usados en la TRRC, con la subsecuente pérdida de cantidades significativas de aminoácidos, vitaminas, micro y macroelementos. Es uno de los tópicos contemplados en el concepto de dialitrauma o pérdidas involuntarias con la TRR ${ }^{67}$.

- Estas pérdidas son proporcionales a la dosis 0 intensidad de la TRRC, y no guardan relación con la técnica de depuración extracorpórea ${ }^{64,66}$.

- No se encuentran diferencias significativas entre las técnicas basadas en difusión y convección, ni tampoco en la capacidad adsortiva o el punto de corte de los filtros para la pérdida de nutrientes durante la TRRC.

- Los requerimientos de energía de los pacientes de CC dependen de su estado nutricional, severidad de la enfermedad y estrés metabólico. Usualmente se presenta hipercatabolismo en estos pacientes, que se caracteriza por una respuesta neurohormonal incrementada frente al estrés metabólico ${ }^{66,68}$.

- Dicho hipercatabolismo se caracteriza por aumento de la expresión de hormonas como adrenalina, glucógeno y cortisol, además de aumento de resistencia a la insulina e incremento de gluconeogénesis ${ }^{64,68}$.

- Lleva a depleción rápida de las reservas de glucosa y glucógeno muscular y hepático, lo que conlleva un consumo de proteínas de origen muscular en cerca del $1 \%$ de proteínas de músculo esquelético cada día.

- Se debe evitar el exceso de aporte energético en estados catabólicos por los riesgos derivados de hiperglucemia, hipertrigliceridemia e hipercapnia con aumento del trabajo respiratorio, acidosis metabólica, esteatosis hepática o sobrehidratación $n^{64}$.

- En los estados de convalecencia, los requerimientos de energía aumentan, excediendo los valores premórbidos, lo cual es relevante como determinante de nutrición insuficiente en fase anabólica, lo que puede retrasar la mejoría del paciente ${ }^{64}$.

Algunas de las guías de nutrición internacionales sugieren el uso de calorimetría indirecta como el estándar de oro para estimar los requerimientos energéticos en CC. El estudio TICACOS (The tight calorie control study) sugiere su superioridad frente a estimaciones basadas en fórmulas. En los pacientes manejados nutricionalmente de acuerdo con fórmulas hubo más complicaciones y mayor tiempo de estancia en $\mathrm{UCl}$, sin embargo, a pesar de la superioridad del uso 
de la calorimetría indirecta, esta no se hace de manera usual en las $\mathrm{UCl}^{68}$.

Dada la pérdida de calor con el uso de TRRC, habitualmente los líquidos administrados a los pacientes son calentados, lo que produce liberación de $\mathrm{CO}_{2}$, aumentando la concentración espiratoria de $\mathrm{CO}_{2}$, lo que falsea los resultados.

Sin embargo, si los líquidos se administran fríos, se incrementan los requerimientos de energía hasta en $1,000 \mathrm{kcal} / \mathrm{d}$ derivados del inicio de termogénesis y aumento de requerimientos de oxígeno ${ }^{64}$.

Los métodos más frecuentemente empleados para la estimación de los requerimientos energéticos en $\mathrm{UCl}$ se basan en el peso ideal. La meta de kilocalorías calculadas para el mismo debe ser de $25-35 \mathrm{kcal} / \mathrm{kg} / \mathrm{d}$ mientras que la suplencia de energía en la fase inicial de catabolismo debe ser de cerca del $30 \%$ del valor obtenido.

El cálculo de requerimientos de energía basado en la ecuación de Harris y Benedict debe corregirse con un coeficiente adecuado que depende, entre otras cosas del uso de TRRC.

Cabe recordar que las estimaciones de requerimientos energéticos son afectadas por el uso de citrato como estrategia de preservación del circuito extracorpóreo (5 mmol de citrato metabolizado proporcionan $3 \mathrm{kcal})$. Cuando se emplean soluciones de diálisis de citrato como buffer (solución tampón), la suplencia de energía no pasa de las $200 \mathrm{kcal} / \mathrm{d}$.

De acuerdo con varios autores, las metas de calorías deben ser cercanas a $25-35 \mathrm{kcal} / \mathrm{kg}$ de peso ideal/día. El sustrato energético sugerido es del $60 \%$ de carbohidratos y el $40 \%$ de triglicéridos; sin embargo, hay trabajos recientes que sugieren dosis más bajas (15- $20 \mathrm{kcal} / \mathrm{kg} / \mathrm{d})$ que no han tenido efectos nocivos ni desenlaces peores, pero han demostrado disminuciones de los requerimientos de insulina con normoglucemia. Sin embargo, la nutrición hipocalórica debe estar basada en un adecuado aporte de proteínas (al menos $1,5 \mathrm{~g} / \mathrm{kg}$ de peso ideal $/ \mathrm{d}$ ) ${ }^{64}$.

\section{Requerimientos de aminoácidos durante la terapia de reemplazo renal continuo}

Los pacientes en UCI suelen tener estados catabólicos que llevan a pérdida de masa muscular como respuesta a la rápida depleción energética.

Así, durante la fase aguda de la enfermedad los aminoácidos se convierten en fuente de glucosa en gluconeogénesis. Dicha pérdida de aminoácidos puede alcanzar de 1,3 a 1,8 $\mathrm{g} / \mathrm{kg}$ de peso ideal/día.
Adicionalmente en los estados de enfermedad crítica hay alteración de la síntesis y metabolismo de aminoácidos esenciales. Además, por su tamaño molecular, son depurados por las TRRC, de hecho, la tirosina es el aminoácido que más se pierde (hasta un $87 \%)^{69}$.

En pacientes críticamente enfermos, se debe considerar un aumento del aporte de proteínas. Se propone que valores mayores de $1,5 \mathrm{~g} / \mathrm{kg}$ de peso ideal/ día pueden mejorar el pronóstico, por lo que guías como la ESPEN así lo recomiendan. De igual manera, cuando se inicia apoyo con TRRC, dicho incremento se recomienda en $0,2 \mathrm{~g} / \mathrm{kg}$ peso ideal $/ \mathrm{dí}^{70,71}$.

\section{Requerimientos de emulsión de lípidos durante la terapia de reemplazo renal continuo}

En la LRA la actividad de la lipasa hepática y la lipólisis están disminuidas, lo que lleva a aumento de contenido de triglicéridos, especialmente en pacientes que reciben alimentación parenteral.

Las TRRC no afectan directamente a los lípidos, ya que su peso molecular impide su permeación a través de las membranas, sean para terapias convectivas o de difusión.

Se recomienda medir periódicamente los triglicéridos para ajustar el aporte de lípidos por nutrición, ya que la acumulación de lípidos puede disminuir la utilidad de las TRRC al producir oclusión de los capilares del filtro, sobre todo cuando se usa heparina como terapia de preservación del circuito. Dicha tendencia es menor con el uso de citrato ${ }^{72}$.

Los efectos de los ácidos grasos insaturados en el funcionamiento del sistema inmunitario dependen de la localización de su doble enlace en las moléculas: los omega 3 se asocian con efectos antiinflamatorios y los omega 6 con impacto proinflamatorio, mientras que los omega 9 se describen como neutros inmunológicamente. Sin embargo, no hay suficiente evidencia para recomendar la prescripción específica de algún subtipo de ácido graso en pacientes en $\mathrm{UCl}^{64}$.

\section{Electrólitos}

La frecuencia de hipopotasemia en pacientes en TRRC oscila entre el 5 y el $25 \%$ de la población, debido al uso de soluciones bajas en potasio sumadas a la depuración de potasio por el sistema extracorpóreo.

En casos de hipopotasemia con $\mathrm{K}$ menor de $3 \mathrm{mEq} / \mathrm{l}$, el riesgo de fibrilación ventricular aumenta y con ello el riesgo de mortalidad. Por tanto, se sugiere 
Gaceta Médica de México. 2018;Supp 1

Tabla 4. Resumen de recomendaciones nutricionales en TRRC (adaptado de Onichimowski, et al., 201764)

\begin{tabular}{|c|c|c|}
\hline Componente & Recomendación & Comentario \\
\hline Energía & $\begin{array}{l}20-25 \mathrm{kcal} / \mathrm{kg} \text { peso ideal } / \mathrm{d} \text { en fase catabólica } \\
25-35 \mathrm{kcal} / \mathrm{kg} \text { peso ideal/d en fase anabólica }\end{array}$ & \\
\hline Glucosa & $4 \mathrm{~g} / \mathrm{kg}$ peso ideal & TRRC no implica ajuste de aporte de carbohidratos \\
\hline Grasas & $0.7-1.5 \mathrm{~g} / \mathrm{kg}$ peso ideal & $\begin{array}{l}\text { No previene su acumulación } \\
\text { No se depuran por TRRC }\end{array}$ \\
\hline Proteínas & $1.7-2.0 \mathrm{~g} / \mathrm{kg}$ peso ideal & $\begin{array}{l}\text { Aumento de } 0,2 \mathrm{~g} / \mathrm{kg} \text { peso ideal comparado con pacientes sin } \\
\text { TRRC }\end{array}$ \\
\hline Sodio & $1,1-1,4 \mathrm{mmol} / \mathrm{kg}$ peso ideal & Evitar cambios rápidos de natremia \\
\hline Potasio & 0,9-2,1 mmol/kg peso ideal & Se recomienda el uso de líquidos de potasio normal \\
\hline Fósforo & $\begin{array}{l}0.2-0.4 \mathrm{mmol} / \mathrm{kg} \text { peso ideal (parenteral) } \\
0.3-0.6 \mathrm{mmol} / \mathrm{kg} \text { peso ideal (enteral) }\end{array}$ & Se recomienda aporte de sustitución \\
\hline Magnesio & $\begin{array}{l}0.1-0.2 \mathrm{mmol} / \mathrm{kg} \text { peso ideal (parenteral) } \\
0.15-0.25 \mathrm{mmol} / \mathrm{kg} \text { peso ideal (enteral) }\end{array}$ & \\
\hline Calcio & $\begin{array}{l}0,4-0,8 \mathrm{mmol} / \mathrm{kg} \text { peso ideal (parenteral) } \\
4-8 \mathrm{mmol} / \mathrm{kg} \text { peso ideal (enteral) }\end{array}$ & Sustitución de calcio especialmente en uso de citrato \\
\hline $\begin{array}{l}\text { Vitaminas } \\
\text { hidrosolubles }\end{array}$ & $\begin{array}{l}\text { B1 } 100 \mathrm{mg} \\
\text { B2 } 2 \mathrm{mg} \\
\text { B3 } 20 \mathrm{mg} \\
\text { B5 } 10 \mathrm{mg} \\
\text { B6 } 100 \mathrm{mg} \\
\text { Biotina (B7) 200 } \mu \mathrm{g} \\
\text { Ácido fólico (B9) } 1 \mathrm{mg} \\
\text { B12 } 4 \mu \mathrm{g} \\
\text { C } 250 \mathrm{mg}\end{array}$ & $\begin{array}{l}\text { Se recomienda el uso del doble de las concentraciones de nutrición } \\
\text { enteral }\end{array}$ \\
\hline Vitaminas liposolubles & $\begin{array}{l}\text { E } 10 \mathrm{IU} \\
\text { K } 4 \text { mg semana }\end{array}$ & \\
\hline
\end{tabular}

el uso de soluciones de potasio normal y restringir el uso de las soluciones libres de potasio solo a casos de hiperpotasemia que comprometa la vida.

El contenido de sodio en los líquidos de sustitución empleados en las TRRC es de $140 \mathrm{mEq} / \mathrm{l}$, por tanto, las TRRC ejercen efecto estabilizador de natremia. En pacientes con hipo o hipernatremia, la TRRC tiende a normalizar el sodio dependiendo de la intensidad de la terapia, lo que puede resultar deletéreo en casos de disnatremias agudas que se corrigen muy rápidamente.

La tasa de cambio de sodio plasmático no debe exceder los $0,5 \mathrm{mmol} / / / \mathrm{h}, 2 \mathrm{mmol} / / / 6 \mathrm{~h}$ y $8 \mathrm{mmol} / / / \mathrm{d}$, por lo que la intensidad de la TRRC y la concentración de sodio se debe ajustar de manera dinámica para evitar modificaciones más rápidas. Así mismo, se debe procurar la administración parenteral de líquidos con sodio ajustados a cada escenario de natremia o modificar las concentraciones de sodio en las bolsas de líquido de sustitución.

La incidencia de hipofosfatemia durante TRRC es descrita clásicamente como alta entre 10,9 y $65 \%$.
Desde que los líquidos de TRRC contienen concentraciones fisiológicas de fosfato, dicha incidencia ha disminuido.

La hipomagnesemia es relativamente rara durante la TRRC y es más común con el uso de soluciones a base de citrato, que se une al calcio y al magnesio, permitiendo eliminarlos a través del filtro. Por ello, las preparaciones disponibles de líquidos de sustitución con citrato tienen concentraciones más altas de magnesio.

La hipocalcemia es frecuente en TRRC, se ha descrito que puede estar presente en hasta el $50 \%$ de los casos. Esto se hace más relevante en los pacientes que reciben soluciones de reposición a base de citrato o lo usan como solución de preservación del circuito extracorpóreo, sin embargo, la hipocalcemia es de fácil corrección y no suele tener repercusiones clínicas cuando se cumple de manera juiciosa con la reposición de calcio post filtro para antagonizar el efecto del citrato. 


\section{Vitaminas}

Las vitaminas hidrosolubles se pierden extensamente durante la TRRC, por lo que guías como la ESPEN hacen recomendaciones para su suplementación (ver tabla 4) ${ }^{70,73}$.

Si bien las vitaminas liposolubles no se eliminan en gran cantidad por los filtros de TRRC, se recomienda su suplementación, excepto de vitamina $A$, en las dosis que se resumen en la tabla $4^{70}$.

\section{Agradecimientos}

Al doctor Camilo Alberto Gonzáles por su apoyo y aportes a la construcción conjunta de los aspectos farmacocinéticos de antibioticoterapia del paciente en TRRC expuestos en el documento.

\section{Bibliografía}

1. Goldstein SL, Nolin TD. Lack of drug dosing guidelines for critically ill patients receiving continuous renal replacement therapy. Clin Pharmacol Ther. 2014;96(2):159-61.

2. Hoste EA, Schurgers M. Epidemiology of acute kidney injury: how big is the problem? Crit Care Med. 2008;36(Suppl 4):S146-51.

3. Kollef $\mathrm{MH}$. Inadequate antimicrobial treatment: an important determinant of outcome for hospitalized patients. Clin Infect Dis. 2000;31(Suppl 4):S131-8.

4. Kollef MH, Sherman G, Ward S, Fraser VJ. Inadequate antimicrobial treatment of infections: a risk factor for hospital mortality among critically ill patients. Chest. 1999;115(2):462-74.

5. Lipman J, Udy AA, Roberts JA. Do we understand the impact of altered physiology, consequent interventions and resultant clinical scenarios in the intensive care unit? The antibiotic story. Anaesth Intensive Care. 2011;39(6):999-1000

6. Tsai D, Lipman J, Roberts JA. Pharmacokinetic/pharmacodynamic considerations for the optimization of antimicrobial delivery in the critically ill. Curr Opin Crit Care. 2015;21(5):412-20.

7. Jamal JA, Mueller BA, Choi GY, Lipman J, Roberts JA. How can we ensure effective antibiotic dosing in critically ill patients receiving different types of renal replacement therapy? Diagn Microbiol Infect Dis. 2015;82(1):92-103

8. Choi G, Gomersall CD, Tian Q, Joynt GM, Li AM, Lipman J. Principles of antibacterial dosing in continuous renal replacement therapy. Blood Purif. 2010;30(3):195-212.

9. Owens RC Jr, Shorr AF. Rational dosing of antimicrobial agents: pharmacokinetic and pharmacodynamic strategies. Am J Health Syst Pharm. 2009:66(12 Suppl 4):S23-30.

10. Heintz BH, Matzke GR, Dager WE. Antimicrobial dosing concepts and recommendations for critically ill adult patients receiving continuous renal replacement therapy or intermittent hemodialysis. Pharmacotherapy. 2009;29(5):562-77.

11. Ambrose PG, Bhavnani SM, Rubino CM, Louie A, Gumbo T, Forrest A, et al. Pharmacokinetics-pharmacodynamics of antimicrobial therapy: it's not just for mice anymore. Clin Infect Dis. 2007;44(1):79-86.

12. Quintiliani R Sr, Quintiliani R Jr. Pharmacokinetics/pharmacodynamics for critical care clinicians. Crit Care Clin. 2008;24(2):335-48.

13. Boucher BA, Wood GC, Swanson JM. Pharmacokinetic changes in critical illness. Crit Care Clin. 2006;22(2):255-71.

14. Eyler RF, Mueller BA; Medscape. Antibiotic dosing in critically ill patients with acute kidney injury. Nat Rev Nephrol. 2011;7(4):226-35

15. Okabe $H$, Mizukami A, Taguchi M, Aiba T, Yasuhara M, Hashimoto $Y$ The increased intestinal absorption rate is responsible for the reduced hepatic first-pass extraction of propranolol in rats with cisplatin-induced renal dysfunction. J Pharm Pharmacol. 2003:55(4):479-86.

16. Wright DH, Pietz SL, Konstantinides FN, Rotschafer JC. Decreased in vitro fluoroquinolone concentrations after admixture with an enteral feeding formulation. JPEN J Parenter Enteral Nutr. 2000;24(1):42-8.

17. Tsai D, Lipman J, Roberts JA. Pharmacokinetic/pharmacodynamic considerations for the optimization of antimicrobial delivery in the critically ill. Curr Opin Crit Care. 2015;21(5):412-20.

18. Prowle JR, Echeverri JE, Ligabo EV, Ronco C, Bellomo R. Fluid balance and acute kidney injury. Nat Rev Nephrol. 2010;6(2):107-15.
19. Udy AA, Roberts JA, Lipman J. Clinical implications of antibiotic pharmacokinetic principles in the critically ill. Intensive Care Med. 2013;39(12):2070-82.

20. Pai MP, Bearden DT. Antimicrobial dosing considerations in obese adult patients. Pharmacotherapy. 2007;27(8):1081-91.

21. Falagas ME, Karageorgopoulos DE. Adjustment of dosing of antimicrobial agents for body weight in adults. Lancet. 2010;375(9710):248-51.

22. Cheatham SC, Fleming MR, Healy DP, Chung CE, Shea KM, Humphrey ML, et al. Steady-state pharmacokinetics and pharmacodynamics of piperacillin and tazobactam administered by prolonged infusion in obese patients. Int J Antimicrob Agents. 2013;41(1):52-6

23. Roberts JA, Lipman J. Optimal doripenem dosing simulations in critically ill nosocomial pneumonia patients with obesity, augmented renal clearance, and decreased bacterial susceptibility. Crit Care Med. 2013;41(2):489-95.

24. Jamal JA, Economou CJ, Lipman J, Roberts JA. Improving antibiotic dosing in special situations in the ICU: burns, renal replacement therapy and extracorporeal membrane oxygenation. Curr Opin Crit Care. 2012:18(5):460-71.

25. Krishnan V, Murray P. Pharmacologic issues in the critically ill. Clin Chest Med. 2003;24(4):671-88

26. Occhipinti DJ, Pendland SL, Schoonover LL, Rypins EB, Danziger LH, Rodvold KA. Pharmacokinetics and pharmacodynamics of two multiple-dose piperacillin-tazobactam regimens. Antimicrob Agents Chemother. 1997;41(11):2511-7.

27. van der Werf TS, Mulder PO, Zijlstra JG, Uges DR, Stegeman CA. Pharmacokinetics of piperacillin and tazobactam in critically ill patients with renal failure, treated with continuous veno-venous hemofiltration (CVVH). Intensive Care Med. 1997;23(8):873-7.

28. Nilsson-Ehle I, Hutchison M, Haworth SJ, Norrby SR. Pharmacokinetics of meropenem compared to imipenem-cilastatin in young, healthy males. Eur J Clin Microbiol Infect Dis. 1991;10(2):85-8.

29. Giles LJ, Jennings AC, Thomson AH, Creed G, Beale RJ, McLuckie A. Pharmacokinetics of meropenem in intensive care unit patients receiving continuous veno-venous hemofiltration or hemodiafiltration. Crit Care Med. 2000;28(3):632-7.

30. Lode H, Grunert K, Koeppe P, Langmaack H. Pharmacokinetic and clinical studies with amikacin, a new aminoglycoside antibiotic. J Infect Dis. 1976;134 SUPPL: S316-22.

31. Kinowski JM, de la Coussaye JE, Bressolle F, Fabre D, Saissi G, Bouvet $O$, et al. Multiple-dose pharmacokinetics of amikacin and ceftazidime in critically ill patients with septic multiple-organ failure during intermittent hemofiltration. Antimicrob Agents Chemother. 1993;37(3):464-73.

32. Blouin RA, Bauer LA, Miller DD, Record KE, Griffen WO Jr. Vancomycin pharmacokinetics in normal and morbidly obese subjects. Antimicrob Agents Chemother. 1982;21(4):575-80.

33. DelDot ME, Lipman J, Tett SE. Vancomycin pharmacokinetics in critically ill patients receiving continuous venovenous haemodiafiltration. $\mathrm{Br} \mathrm{J}$ Clin Pharmacol. 2004;58(3):259-68.

34. Benvenuto M, Benziger DP, Yankelev S, Vigliani G. Pharmacokinetics and tolerability of daptomycin at doses up to 12 milligrams per kilogram of bodyweight once daily in healthy volunteers. Antimicrob Agents Chemother. 2006;50(10):3245-9.

35. Vilay AM, Grio M, Depestel DD, Sowinski KM, Gao L, Heung M, et al. Daptomycin pharmacokinetics in critically ill patients receiving continuous venovenous hemodialysis. Crit Care Med. 2011;39(1):19-25.

36. Roberts JA, Pea F, Lipman J. The clinical relevance of plasma protein binding changes. Clin Pharmacokinet. 2013;52(1):1-8.

37. Pea F, Viale P, Furlanut M. Antimicrobial therapy in critically ill patients: a review of pathophysiological conditions responsible for altered disposition and pharmacokinetic variability. Clin Pharmacokinet. 2005;44(10):1009-34.

38. Pea F, Viale P, Pavan F, Furlanut M. Pharmacokinetic considerations for antimicrobial therapy in patients receiving renal replacement therapy. Clin Pharmacokinet. 2007;46(12):997-1038.

39. Maynar J, Sánchez-Izquierdo JA. Dosificación de fármacos durante los tratamientos de depuración extracorpórea de la sangre. Nefro Plus 2010;3:20-6.

40. Scoville BA, Mueller BA. Medication dosing in critically ill patients with acute kidney injury treated with renal replacement therapy. Am J Kidney Dis. 2013;61(3):490-500

41. Roberts DM. The relevance of drug clearance to antibiotic dosing in critically ill patients. Curr Pharm Biotechnol. 2011;12(12):2002-14.

42. Blot S, Lipman J, Roberts DM, Roberts JA. The influence of acute kidney injury on antimicrobial dosing in critically ill patients: are dose reductions always necessary? Diagn Microbiol Infect Dis. 2014;79(1):77-84.

43. Seyler L, Cotton F, Taccone FS, De Backer D, Macours P, Vincent JL, et al. Recommended $\beta$-lactam regimens are inadequate in septic patients treated with continuous renal replacement therapy. Crit Care. 2011;15(3):R137.

44. Roberts DM, Roberts JA, Roberts MS, Liu X, Nair P, Cole L, et al. RENAL Replacement Therapy Study Investigators. Variability of antibiotic concentrations in critically ill patients receiving continuous renal replacement therapy: a multicentre pharmacokinetic study. Crit Care Med. 2012; 40(5):1523-8.

45. Lewis SJ, Mueller BA. Antibiotic dosing in patients with acute kidney injury: "Enough but not too much". J Intensive Care Med. 2016;31(3):164-76. 
46. Echeverri J. Terapia de soporte dialítico agudo. Compendio de terapéutica: evidencia actual. 6. ${ }^{\text {a }}$ edición Editorial médica Celsus; 2017. pp. 403-12.

47. Shaw AR, Mueller BA. Antibiotic dosing in continuous renal replacement therapy. Adv Chronic Kidney Dis. 2017;24(4):219-27.

48. Roberts JA, Choi GY, Joynt GM, Paul SK, Deans R, Peake S, et al. Sampling antibiotics in renal replacement therapy (SMARRT): an observationa pharmacokinetic study in critically ill patients. BMC Infect Dis. 2016;16:103.

49. Roberts JA, Lefrant JY, Lipman J. What's new in pharmacokinetics of antimicrobials in AKI and RRT? Intensive Care Med. 2017;43(6):904-6.

50. Robert R, Rochard E, Malin F, Bouquet S. Amikacin pharmacokinetics during continuous veno-venous hemofiltration. Crit Care Med. 1991;19(4):588-9.

51. Kroh UF, Lennartz H, Edwards DJ, Stoeckel K. Pharmacokinetics of ceftriaxone in patients undergoing continuous veno-venous hemofiltration. J Clin Pharmacol. 1996;36(12):1114-9.

52. Bouman CS, van Kan HJ, Koopmans RP, Korevaar JC, Schultz MJ, Vroom MB. Discrepancies between observed and predicted continuous venovenous hemofiltration removal of antimicrobial agents in critically il patients and the effects on dosing. Intensive Care Med. 2006;32(12):2013-9.

53. Isla A, Gascon AR, Maynar J, Arzuaga A, Toral D, Pedraz JL. Cefepime and continuous renal replacement therapy (CRRT): in vitro permeability of two CRRT membranes and pharmacokinetics in four critically ill patients. Clin Ther. 2005;27(5):599-608.

54. Gilbert B, Robbins P, Livornese LL Jr. Use of antibacterial agents in renal failure. Infect Dis Clin North Am. 2009;23(4):899-924, viii.

55. Cirillo I, Vaccaro N, Balis D, Redman R, Matzke GR. Influence of continuous venovenous hemofiltration and continuous venovenous hemodiafiltration on the disposition of doripenem. Antimicrob Agents Chemother. 2011:55(3):1187-93.

56. Burkhardt O, Hafer C, Langhoff A, Kaever V, Kumar V, Welte T, et al. Pharmacokinetics of ertapenem in critically ill patients with acute renal failure undergoing extended daily dialysis. Nephrol Dial Transplant. 2009:24(1):267-71.

57. Gobernado M, Acuña C. Ertapenem. Rev Esp Quimioter. 2007; 20(3):277-99

58. Muhl $\mathrm{E}$, Martens $\mathrm{T}$, Iven $\mathrm{H}, \mathrm{Rob} \mathrm{P}$, Bruch $\mathrm{HP}$ Influence of continuous veno-venous haemodiafiltration and continuous veno-venous haemofiltration on the pharmacokinetics of fluconazole. Eur J Clin Pharmacol. 2000:56(9-10):671-8.

59. Yagasaki K, Gando S, Matsuda N, Kameue T, Ishitani T, Hirano T, et al. Pharmacokinetics and the most suitable dosing regimen of fluconazole in critically ill patients receiving continuous hemodiafiltration. Intensive Care Med. 2003;29(10):1844-8.
60. Fish DN, Teitelbaum I, Abraham E. Pharmacokinetics and pharmacodynamics of imipenem during continuous renal replacement therapy in critically ill patients. Antimicrob Agents Chemother. 2005;49(6):2421-8.

61. Mendes CA, Burdmann EA. Polymyxins - review with emphasis on nephrotoxicity. Rev Assoc Med Bras. 2009;55(6):752-9.

62. Curkovic I, Luthi B, Franzen D, Ceschi A, Rudiger A, Corti N. Trimethoprim/ sulfamethoxazole pharmacokinetics in two patients undergoing continuous venovenous hemodiafiltration. Ann Pharmacother. 2010;44(10):1669-72.

63. Bendavid I, Singer P, Theilla M, Themessl-Huber M, Sulz I, Mouhieddine M,Schuh C, et al. Nutrition day ICU: A 7 year worldwide prevalence study of nutrition practice in intensive care. Clin Nutr. 2017;36(4):1122-9.

64. Onichimowski D, Goraj R, Jalali R, Grabala J, Mayzner-Zawadzka E, Czuczwar M. Practical issues of nutrition during continuous renal replacement therapy. Anaesthesiol Intensive Ther. 2017;49(4):309-16.

65. Zusman O, Theilla M, Cohen J, Kagan I, Bendavid I, Singer P. Resting energy expenditure, calorie and protein consumption in critically ill patients: a retrospective cohort study. Crit Care. 2016;20(1):367.

66. Honoré PM, De Waele E, Jacobs R, Mattens S, Rose T, Joannes-Boyau O, et al. Nutritional and metabolic alterations during continuous renal replacement therapy. Blood Purif. 2013;35(4):279-84.

67. Maynar Moliner J, Honore PM, Sánchez-Izquierdo Riera JA, Herrera Gutiérrez M, Spapen HD. Handling continuous renal replacement therapy-related adverse effects in intensive care unit patients: the dialytrauma concept. Blood Purif. 2012;34(2):177-85.

68. Casaer MP, van den Berghe G. Nutrition in the acute phase of critical illness. N Engl J Med. 2014;370(13):1227-36.

69. Scheinkestel CD, Adams F, Mahony L, Bailey M, Davies AR, Nyulasi I, et al. Impact of increasing parenteral protein loads on amino acid levels and balance in critically ill anuric patients on continuous renal replacement therapy. Nutrition. 2003;19(9):733-40.

70. Cano N, Fiaccadori E, Tesinsky P, Toigo G, Druml W; DGEM (German Society for Nutritional Medicine), Kuhlmann M, Mann H, Hörl WH; ESPEN (European Society forParenteral and Enteral Nutrition). ESPEN Guidelines on enteral nutrition: Adult renal failure. Clin Nutr. 2006;25(2):295-310.

71. Wiesen P, van Overmeire L, Delanaye P, Dubois B, Preiser JC. Nutrition disorders during acute renal failure and renal replacement therapy. JPEN J Parenter Enteral Nutr. 2011;35(2):217-22.

72. Kazory A, Clapp WL, Ejaz AA, Ross EA. Shortened hemofilter survival time due to lipid infusion in continuous renal replacement therapy. Nephron Clin Pract. 2008:108(1):c5-9.

73. Cano NJ, Aparicio M, Brunori G, Carrero JJ, Cianciaruso B, Fiaccadori E, et al. ESPEN. ESPEN Guidelines on parenteral nutrition: adult renal failure. Clin Nutr. 2009;28(4):401-14. 